\title{
Candida-reactive T cells for diagnosis of invasive Candida infection
}

Felix C. Koehler ${ }^{1}$, Oliver A. Cornely ${ }^{1,2,3}$, Hilmar Wisplinghoff ${ }^{4,5,6}$, Astrid C. Schauss ${ }^{1}$, Jon Salmanton-Garcia², Helmut Ostermann ${ }^{7}$, Maren Ziegler $^{4}$, Petra Bacher ${ }^{8}$, Alexander Scheffold ${ }^{8,9}$, Regina Alex ${ }^{10}$, Anne Richter ${ }^{10}$, Philipp Koehler ${ }^{1,2}$

${ }^{1}$ Cologne Excellence Cluster on Cellular Stress Responses in Aging-Associated Diseases (CECAD), University of Cologne, Cologne, Germany ${ }^{2}$ Department I of Internal Medicine, ECMM Diamond Center of Excellence in Medical Mycology, German Centre for Infection Research (DZIF), University of Cologne, Cologne, Germany ${ }^{3}$ Clinical Trials Centre Cologne (ZKS Köln), University of Cologne, Cologne, Germany ${ }^{4}$ Labor Dr. Wisplinghoff, Cologne, Germany ${ }^{5}$ Institute for Medical Microbiology, Immunology and Hygiene, University of Cologne, Cologne, Germany ${ }^{6}$ Institute for Clinical Microbiology, University Witten/Herdecke, Witten, Germany ${ }^{7}$ Department of Internal Medicine III, University of Munich, Munich, Germany ${ }^{8}$ Department of Cellular Immunology, Clinic for Rheumatology and Clinical Immunology, Charité - University Medicine Berlin, Berlin, Germany ${ }^{9}$ German Rheumatism Research Centre (DRFZ) Berlin and Leibniz Association, Berlin, Germany ${ }^{10}$ Miltenyi Biotec GmbH, Bergisch Gladbach, Germany

\section{Objectives}

Invasive Candida infection $(\mathrm{ICl})$ is the most common fungal bloodstream infection. Blood and tissue cultures are the current gold standard diagnostic methods, however false-negatives remain a clinical challenge. Tissue culture and histology burden certain risks for the patient and are often contraindicated due to underlying medical conditions. Candida-reactive $T$ cells were quantitated based on the upregulation of CD69/CD154 (CD40L) from peripheral blood as new diagnostic read-out for ICl. In a pilot study, we examined healthy donors and three patient cohorts with either proven $\mathrm{ICl}$, suspected $\mathrm{ICl}$, or high risk of $\mathrm{ICl}$.

Methods

Candida cells were lysed mechanically by gentleMACS ${ }^{\circledR}$ dissociator. Peripheral blood mononuclear cells (PBMC) of patients and healthy donors were isolated by density gravitation. PBMCs were stained with 7AAD (Miltenyi Biotec $\mathrm{GmbH}$, Germany and eBioscience, San Diego, CA, USA) and count of viable cells was examined on a MACSQuant ${ }^{\circledR}$ flow cytometer (all Miltenyi Biotec $\mathrm{GmbH}$, Germany). $1 \times 10^{6}$ cells $/ 100 \mu \mathrm{l}$ and well were seeded into 96 well flat-bottom plates. PBMCs were incubated overnight at $37^{\circ} \mathrm{C}$ with $5 \% \mathrm{CO}_{2}$. Cultured cells were stimulated with $\mathrm{CD} 28$ and $\mathrm{CD} 40$ pure antibodies and co-incubated with lysate of either $\mathrm{C}$. albicans, $\mathrm{C}$. glabrata, $\mathrm{C}$. parapsilosis, $C$. tropicalis or $\mathrm{C}$. krusei for $5 \mathrm{~h}$ in $5 \% \mathrm{CO}_{2}$. Each Candida spp. resulted in a separate stimulation. Missing challenge with fungal lysate served as negative control, Staphylococcal Enterotoxin B (SEB) as positive control and cells were analyzed on a MACSQuant ${ }^{\circledR}$ flow cytometer.

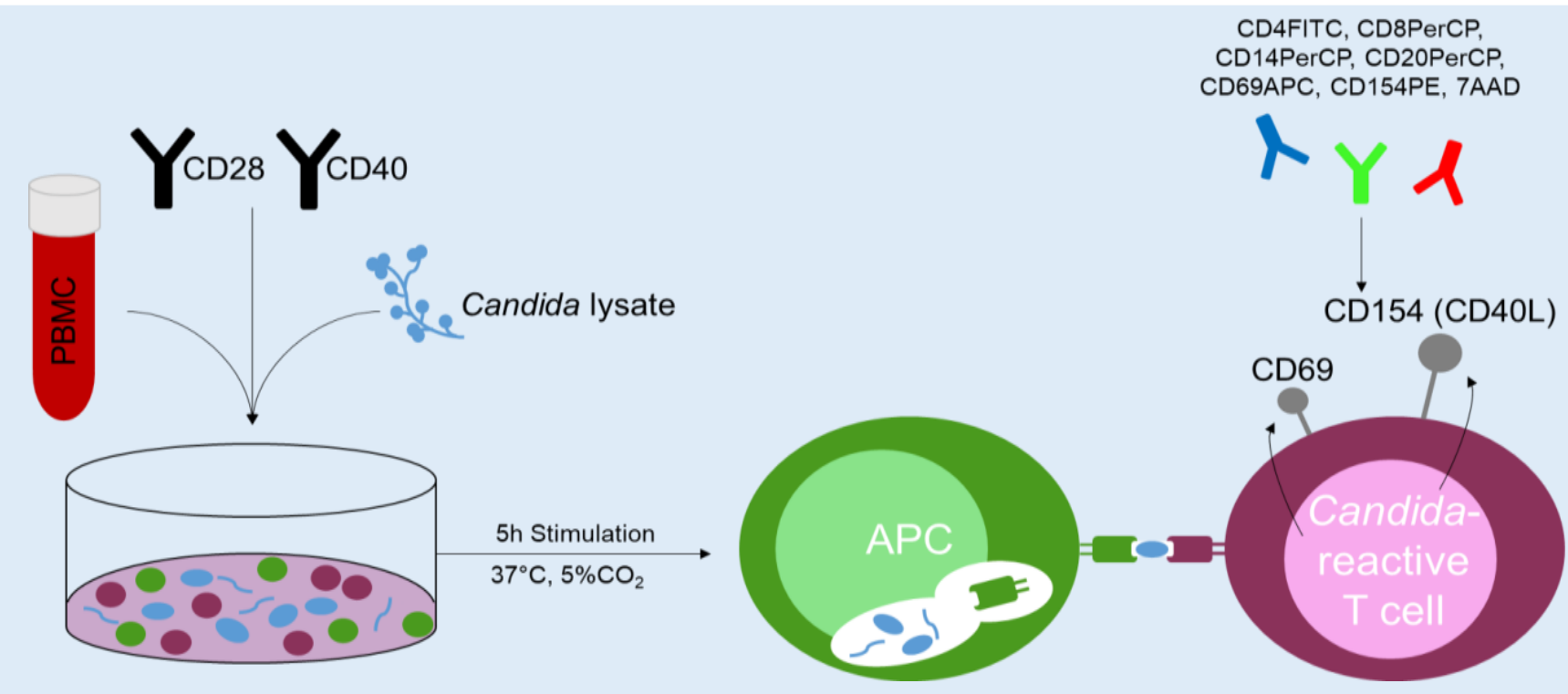

Figure 1. CD69/CD154 (CD40L) upregulation for measurement of Candida-reactive T cells.

A
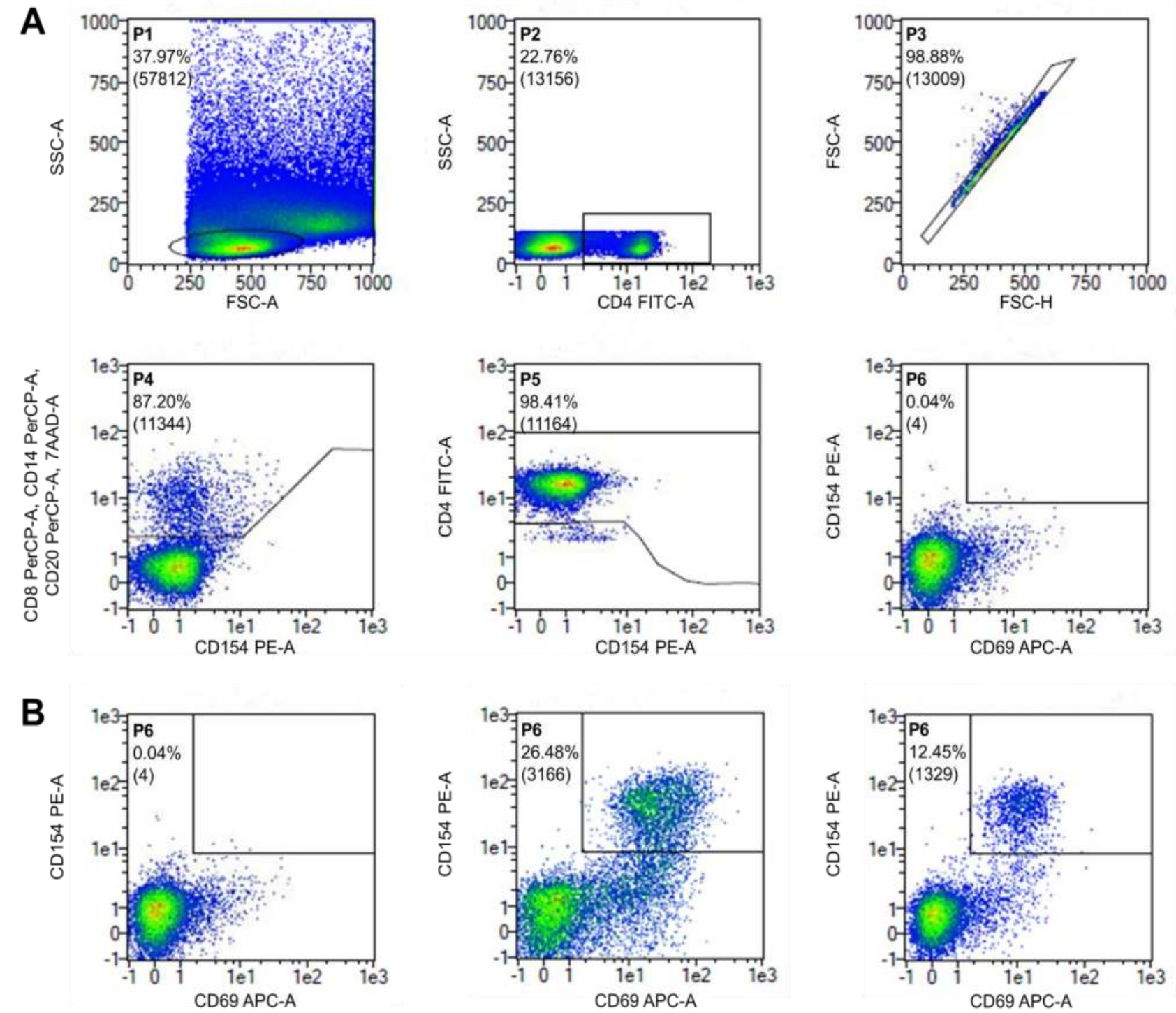

Figure 2. Flow Cytometry - Gating Strategy and detection of Candida albicans-reactive T cells. Cell frequency (\%) and absolute cell (n) count. (A) Gating strategy. Negative control. (B) Detection of Candida-reactive T cells. From left to right. Negative control; positive control; antigen-stimulated probe.

\section{Statistics}

Cut-off values discriminating between healthy donors, disease control and patients with proven $\mathrm{ICl}$ were calculated by receiver operating characteristic analysis using IBM SPSS Statistics software (Version 23, IBM Corporation, NY, USA). For clinical evaluation, patient cohorts were grouped according to the 2008 European Organisation for Research and Treatment of Cancer/Invasive Fungal Infections Cooperative Group and the National Institute of Allergy and Infectious Diseases Mycoses Study Group (EORTC/MSG) criteria.

Results

In a prospective pilot study, we determined the performance of the Candida-reactive lymphocyte assay in 26 patients, including 16 proven $\mathrm{ICl}$ and one patient with probable hepatosplenic candidiasis. Nine hematological high-risk patients served as disease control and 14 healthy donors as negative control. To examine the mean frequency and to calculate the cut-off value of $C$. albicans-reactive $T$ cells in healthy individuals we included an additional cohort of 96 healthy blood donors.

Thirteen of 16 patients with proven $\mathrm{ICl}$ and one patient with probable $\mathrm{ICl}$ had elevated levels of Candida-reactive $\mathrm{CD}^{+} \mathrm{T}$ cells. Due to autofluorescence of cells we excluded three candidemia patients from analysis. In 10 of 12 proven $\mathrm{ICI}$, T cell reaction matched the Candida spp. identified by conventional diagnostics. One histology proven $\mathrm{ICl}$ patient had no species identification by standard diagnostics.

Disease and healthy control patients of the pilot study cohort had no elevated Candida-directed T cells counts.
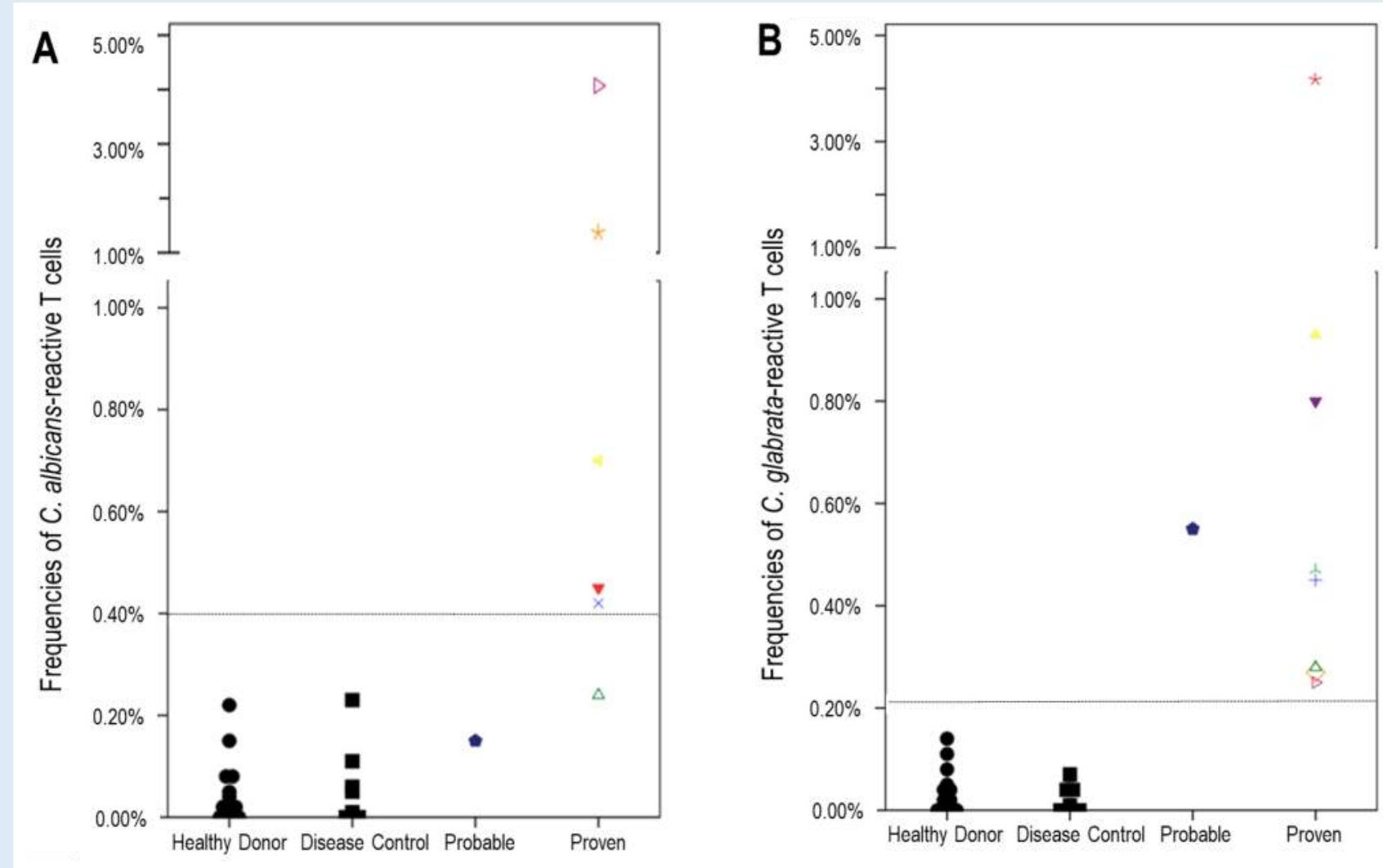

Figure 3. Frequencies of Candida-reactive T cells in healthy donors, disease control and patients with probable or proven ICI. Test positivity considered elevated levels of Candidareactive $T$ cells with simultaneously 3.05 fold increase of antigen-stimulated $T$ cells compared to unstimulated T cells. Dashed lines show cut-off values for Candida albicans-reactive T cells $(0.40 \%)$ and Candida glabrata-reactive T cells $(0.22 \%)$. (A) Frequencies of Candida albicans $\mathrm{CD}^{+} 9^{+} \mathrm{CD} 154^{+} \mathrm{T}$ cells among $\mathrm{CD4} 4^{+} \mathrm{T}$ cells. (B) Frequencies of Candida glabrata

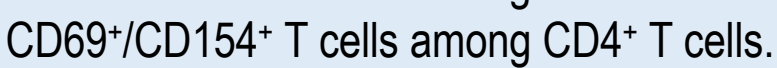

\section{Conclusion}

The Candida-reactive lymphocyte assay correctly identified the majority of $\mathrm{ICl}$ patients by species level. Autofluorescence of cells and insufficient cell count of T cells are limiting factors. The Candida-reactive lymphocyte assay has the potential to complement current diagnostic assays for invasive Candida infection, especially when blood cultures remain negative and when tissue cultures and histopathology are contraindicated.

\section{Acknowledgments}

Mario Assenmacher, Elisa Barth, Denis Hoffmann, Larisa Idrizovic, Stefan Körfgen, Tatjana Lammertz and Julia Milleck. 